\title{
An integrated two-stage process for effective dechlorination of polychlorinated biphenyls in subcritical water in the presence of hydrogen donors
}

\author{
Neng-Min Zhu, Chun-Feng Wang, Fu-Shen Zhang* \\ Research Center For Eco-Environmental Sciences, Chinese Academy of Sciences, 18 Shuangqing Road, Beijing 100085, China
}

\section{H I G H L I G H T S}

- Corrosion of $\mathrm{Fe}^{0}$ and dechlorination are conducted at different temperature, respectively.

- Corrosion of $\mathrm{Fe}^{\mathrm{O}}$ conducted at $150^{\circ} \mathrm{C}$ favors product of reactive hydrogen species.

- Slight addition of oxalic acid enhances utility efficiency of $\mathrm{Fe}^{0}$ without dislodgment of $\mathrm{Ni}$ from $\mathrm{Ni} / \mathrm{Fe}$.

\section{G R A P H I C A L A B S T R A C T}

Effect of residence time on dechlorination during two-stage reaction process. $X$ axis: residence time at first stag, $C_{\mathrm{PCBs}}=5.25 \mathrm{mg} / \mathrm{L}, C_{\mathrm{Ni} / \mathrm{Fe}(\mathrm{s})}=6.25 \mathrm{~g} / \mathrm{L}, T_{\text {first stage }}=150{ }^{\circ} \mathrm{C}, T_{\text {second stage }}=330^{\circ} \mathrm{C}$.

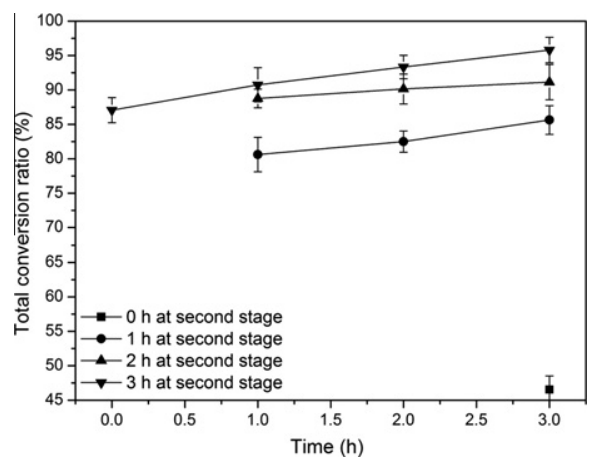

\begin{abstract}
A B S T R A C T
In this study, a novel two-stage process, i.e. first stage for hydrogen species generation at $150{ }^{\circ} \mathrm{C}$ and second stage for $\mathrm{PCB}$ dechlorination at $330^{\circ} \mathrm{C}$, was developed for efficient PCB degradation in subcritical water with nanoscale $\mathrm{Ni} / \mathrm{Fe}$ particles. The obtained results showed that over $92 \%$ of PCBs was completely converted into 1-alkyl-benzenes during this process. The addition of hydrogen donors could greatly enhance PCB dechlorination in descending order: oxalic acid $\approx \mathrm{HAC}>\mathrm{NaH}_{2} \mathrm{PO}_{4} \approx \mathrm{KH}_{2} \mathrm{PO}_{4}$. The anion introduced along with $\mathrm{H}^{+}$played the crucial role in overall dechlorination process due to the undesired reaction between anion and reactive nanoscale $\mathrm{Ni} / \mathrm{Fe}$. Oxalic acid was quite effective for the generation of hydrogen species by supply more available $\mathrm{H}^{+}$without undesired side reaction. In contrast, phosphate could react with $\mathrm{Ni} / \mathrm{Fe}$ rapidly to form complex precipitates on particle surface and thereby block the reactive sites of particle following suspension of dechlorination. In addition, low $150{ }^{\circ} \mathrm{C}$ at first stage as compared to $330^{\circ} \mathrm{C}$ at second stage favored the dissolution and adsorption of hydrogen on Ni surface for catalyzing to form more reactive hydrogen species used for rapid dechlorination.
\end{abstract}

(c) 2012 Elsevier B.V. All rights reserved.

\section{Introduction}

In recent years, reductive dehalogenation with iron-based particles in subcritical water has attracted more attention due to the absence of undesired by-products such as polychlorinated

\footnotetext{
* Corresponding author. Tel./fax: +86 1062849515 .

E-mail address: fszhang@rcees.ac.cn (F.-S. Zhang).
}

dibenzo-p-dioxins and dibenzofurans in this process. As hydrogen donor, water under subcritical state can supply more available $\mathrm{H}_{3} \mathrm{O}^{+}$and energy for accelerating rate constant as compared to ambient water during this process [1-5]. Meanwhile, the solubility of organics is enhanced greatly in subcritical water, and therefore the limits of mass transfer between water phase and organic phase decline remarkably. As electron donor, $\mathrm{Fe}^{0}$ provides electron via corrosion to reduce $\mathrm{H}^{+}$to molecular hydrogen which was used 
for subsequent hydrodehalogenation. Hence, corrosion of $\mathrm{Fe}^{0}$ and generation of hydrogen species are always considered as the key steps during the whole dehalogenation process [6,7]. However, the low utility efficiency of $\mathrm{Fe}^{0}$ due to the low $\mathrm{H}^{+}$concentration and formation of passivation layer always presents as obstacle to effective dehalogenation, especially in the case of complicated composition of PCBs mixture, such as commercial Aroclor $[5,8]$.

Studies have aimed at enhancing utility efficiency of $\mathrm{Fe}^{0}$ by introduction hydrogen donors in order to increase available $\mathrm{H}^{+}$ concentration, such as inorganic and organic acid [9-13]. Meanwhile, addition of acid can also retard the formation of passivation layers on particles surface because the precipitates of iron oxide is dependent on the $\mathrm{pH}$ of water in terms of mechanisms depicted as follows $[6,10,13]$.

$\mathrm{Fe}^{0}+2 \mathrm{H}_{2} \mathrm{O} \rightarrow \mathrm{Fe}^{2+}+\mathrm{H}_{2(\mathrm{~g})}+2 \mathrm{OH}^{-}$

$\mathrm{Fe}^{2+}+2 \mathrm{OH}^{-} \rightarrow \mathrm{Fe}(\mathrm{OH})_{2} \rightarrow \mathrm{FeO}+\mathrm{H}_{2} \mathrm{O}$

$2 \mathrm{FeO}+\mathrm{H}_{2} \mathrm{O} \rightarrow \mathrm{Fe}_{2} \mathrm{O}_{3}+2 \mathrm{H}^{\circ}$

But further discussion about the role of these hydrogen donors at high temperature such as in subcritical water is still scarce.

It should be noted that the high temperature in subcritical water not only favors the dehalogenation but also facilitates the formation of passivation layer. However, batch experiments in previous reports were conducted with improper consideration that the optimum reaction temperature for dehalogenation was also best suitable for corrosion of $\mathrm{Fe}^{0}$ and catalysis of hydrogen species. This ignored that each section reaction involved in whole dehalogenation process, such as dehalogenation, corrosion of $\mathrm{Fe}^{0}$, generation of hydrogen species, has its own optimum reaction conditions. Zhu et al. [4] found that the presence of PCBs could not affect the chemical behavior of $\mathrm{Ni} / \mathrm{Fe}$ particles in subcritical water, implying that corrosion of $\mathrm{Fe}^{0}$ and subsequent generation of hydrogen species are independent of the presence of PCBs. Therefore, corrosion of $\mathrm{Fe}^{0}$ for production of hydrogen species and subsequent dechlorination can be carried out separately in terms of reaction temperature and time in order to optimize each section reaction and thereby enhance overall dechlorination efficiency significantly. It has been reported that the hydrogen species in aqueous phase other than gas phase is used for dechlorination [14]. Hence, corrosion of $\mathrm{Fe}^{0}$ at low temperature other than at high temperature cannot only result more hydrogen species dissolve in aqueous phase because the solubility of hydrogen species in aqueous phase increase as the decrease of temperature in subcritical water but also retard the formation of passivation layer on particle surface.

In present study, the whole dechlorination process was divided into two stages in term of reaction temperature. The corrosion of $\mathrm{Fe}^{0}$ was conducted at first stage below $200{ }^{\circ} \mathrm{C}$ in the both presence of hydrogen donors, and following PCB dechlorination was carried out at second stage as increase of temperature from first stage to $330^{\circ} \mathrm{C}$. The primary objective of this study is to elucidate the effect of hydrogen donor on $\mathrm{Fe}^{0}$ corrosion and generation of hydrogen species at first stage. And the comprehensive influence of hydrogen donor on whole PCB dechlorination under this two-stage process was also investigated.

\section{Experimental}

\subsection{Materials}

PCB-contained capacitor oil was obtained from a certificated PCBs disposal company located in north-east China. The stock solution used for each dechlorination run was prepared by diluting raw capacitor oil with hexane and stored in a refrigerator. Analytical grade chemical reagents involved in this study were: acetic acid, oxalic acid, ethanol, $\mathrm{NaH}_{2} \mathrm{PO}_{4}, \quad \mathrm{KH}_{2} \mathrm{PO}_{4}, \quad \mathrm{FeSO}_{4} \cdot 7 \mathrm{H}_{2} \mathrm{O}, \mathrm{Ni}(-$ $\left.\mathrm{NO}_{3}\right)_{2} \cdot 9 \mathrm{H}_{2} \mathrm{O}, \mathrm{NaOH}, \mathrm{NaBH}_{4}, \mathrm{Na}_{2} \mathrm{SO}_{4}$. HPLC grade n-hexane and acetone were supplied by Jackber Company, USA. Deionized water used in the study was adequately purged with $\mathrm{N}_{2}$ before use to create an anoxic aqueous environment. Arochlor 1242 standards $(1000 \mu \mathrm{g} / \mathrm{mL}$ in hexane) used as calibration standard and PCB209 $(1000 \mu \mathrm{g} / \mathrm{mL}$ in hexane) used as surrogate standard were obtained from AccuStandard, USA.

\subsection{Preparation of bimetallic Ni/Fe nanoparticles}

$\mathrm{Ni} / \mathrm{Fe}$ particles were synthesized in ethanol/water solution by reducing $\mathrm{Fe}^{2+}$ and $\mathrm{Ni}^{2+}$ to $\mathrm{Fe}^{0}$ and $\mathrm{Ni}^{0}$ using $\mathrm{BH}_{4}^{-}$. Detailed preparation and collection procedure were described in previous report [4]. The Ni was selected as the catalytic candidate in bimetallic particles rather than other precious metals such as Pd, Pt, Rh because it is a more cost-effective alternative. The ratio of Fe:Ni was adjusted to 9:1 (w/w) corresponding theoretical 10\% (wt.\%) $\mathrm{Ni}$ in bimetallic particles. The resultant black particles were stored in a sealed container filled with acetone and then placed in a refrigerator. Before each experiment, black particles were dried in vacuum to remove acetone for use.

\subsection{Characterization of $\mathrm{Ni} / \mathrm{Fe}$ particles}

Transmission electron microscope (TEM) images were obtained using a Hitachi H-7500 microscope. X-ray diffraction (XRD) analysis was carried out using Philips PW 1700 X-ray diffractometer. Inductively coupled plasma-optical emission spectrometry (Perkin Elmer OPTIMA 2000, USA) was used to determine the actual Ni:Fe ratios of synthesized $\mathrm{Ni} / \mathrm{Fe}$ samples and concentration of metal ions in aqueous phase after reaction.

\subsection{Batch experiments}

Batch tests were conducted in a $100 \mathrm{~mL}$ autoclave constructed from $316 \mathrm{~L}$ materials. Before experiment, $0.5 \mathrm{~g}$ of vacuum-dry $\mathrm{Ni} /$ Fe particles was placed into the autoclave. Then $1 \mathrm{~mL}\left(420 \mu \mathrm{g} \mathrm{mL}^{-1}\right.$ of PCBs) stock solution of capacitor oil was quickly spiked onto particles directly to form an $840 \mathrm{mg} \mathrm{kg}^{-1}$ PCBs uptake in the particles. In order to protect particles from oxidation in the air, hexane in the autoclave was dried by gentle nitrogen stream. Due to a small amount used, each hydrogen donor was dissolved in $80 \mathrm{~mL}$ deionized water to form homogenous solution for use. After complete evaporation of hexane, $80 \mathrm{~mL}$ homogenous solution containing hydrogen donor was decanted into the autoclave gently to result in a Ni/Fe concentration of $6.25 \mathrm{~g} \mathrm{~L}^{-1}$ and initial PCBs concentration of $5.25 \mathrm{mg} \mathrm{L}^{-1}$. Subsequently, the autoclave was screw down immediately and then pure Ar gas was flushed through interior of the autoclave to discharge the unwanted air. After purge with Ar gas, the sealed autoclave was heated by electrical rod-like heater to a desired temperature and hold for a certain time. After termination of reaction, the hot autoclave was cooled down by electrical fan in atmosphere. All experiments were carried out in triplicate and each data point reported in all figures is mean of triplicates with standard deviation.

\subsection{Extraction, cleanup and analysis procedure}

The extraction, cleanup and analysis procedures were performed according to literatures and EPA methods [4,15-17]. After cooling, the autoclave was opened carefully and the surrogate standard of PCBs 209 was added into the autoclave for quality control. Aqueous phase was subjected to liquid-liquid extraction with hexane. The solid particles were separated from aqueous phase with strong magnet and allowed to air-dry for subsequent ultrasonic 
extraction with mixture of acetone and hexane $(1: 1, v / v)$. Then all organic extracts were combined and concentrated with rotary evaporator to approximate $2 \mathrm{~mL}$ for solvent exchange with hexane. The received hexane phase was concentrated again with rotary evaporator to approximate $1 \mathrm{~mL}$ for concentrated sulfuric acid cleanup in a separatory funnel. After washing the sulfuric acid with deionized water in separatory funnel, the hexane phase passed through a cleanup column consisted of $1 \mathrm{~cm}$ sodium sulfate, and then $5 \mathrm{~cm}$ of PR Grade Florisil (60-100 mesh) topped with $1 \mathrm{~cm}$ of sodium sulfate. Then the cleanup column was eluted with $50 \mathrm{~mL}$ hexane, and the received eluate in a ball flask was concentrated to $1 \mathrm{~mL}$ for GC-MS analysis using an Agilent 7890A gas chromatograph equipped with an Agilent 5975C mass spectrometry and autosampler (USA). Chromatographic separation were accomplished with a HP-5 capillary column $(30 \mathrm{~m} \times 0.25 \mathrm{~mm}$ i.d. $\times 0.25 \mu \mathrm{m}$ film thickness) using splitless injections. The initial oven temperature was set at $50^{\circ} \mathrm{C}$ and ramped to $150{ }^{\circ} \mathrm{C}$ at $20{ }^{\circ} \mathrm{C} \mathrm{min}-1$, then to $200{ }^{\circ} \mathrm{C}$ at $2{ }^{\circ} \mathrm{C} \mathrm{min}^{-1}$, finally to $250{ }^{\circ} \mathrm{C}$ hold for $1 \mathrm{~min}$. Injector and detector temperatures were 280 and $300{ }^{\circ} \mathrm{C}$, respectively. Ultrahigh pure helium was used as the carrier gas at a constant flow rate of $1 \mathrm{~mL} \mathrm{~min}^{-1}$.

Quantification of total PCBs in capacitor oil was based on the calibration with Aroclor 1242 using eight major congeners following EPA method 8270 and previous literatures $[4,16,17]$ due to the equivalence of congener distribution between capacitor oil and Aroclor 1242. The Arabic symbols in order labeled in Fig. 3 denoted the selected PCB peaks for quantification. The work of Frame et al. [18] was used as a reference for composition of the Aroclor 1242. In separate control experiments, the recoveries for PCB 209 were found to be $85-105 \%$, which can meet the accuracy of quantification.

\subsection{Data interpretation}

Total PCB conversion ratio is defined as follows:

Total conversion ratio $(\%)=\frac{c_{o}-c_{\text {product }}}{c_{o}}$

where $c_{o}$ and $c_{\text {product }}$ are PCBs concentration in stock solution of capacitor oil and product mixture, respectively $\left(\mathrm{mg} \mathrm{L}^{-1}\right)$.

\section{Results and discussion}

\subsection{Characterization of $\mathrm{Ni} / \mathrm{Fe}$ particles}

TEM imagine of fresh particles in Fig. 1a shows that particles exhibited an average diameter of $20-80 \mathrm{~nm}$ and formed aggregated structure due to the magnetic dipole interactions [19]. As for spent particles, a slight expansion of particle is observed after reaction in Fig. 1b. This might be ascribed to the buildup of passivation layer on particle surface [20,21].

Fig. 2a presents the XRD patterns of different particles before and after reaction. No characteristic peaks are associated with crystals of $\mathrm{Fe}^{0}$ and $\mathrm{Ni}^{0}$ for fresh $\mathrm{Ni} / \mathrm{Fe}$ particles, which implies that amorphous structure was formed in the synthesis process. Only iron oxides $\left(\mathrm{Fe}_{3} \mathrm{O}_{4} / \gamma-\mathrm{Fe}_{2} \mathrm{O}_{3}\right)$ appeared after reaction in the presence of acetic acid and oxalic acid, resembling that observed in pure subcritical water [4]. This means that the presence of acetic and oxalic acid did not affect the reaction behavior of nanoscale $\mathrm{Ni} / \mathrm{Fe}$ remarkably in subcritical water. In contrast, many characteristic peaks of iron phosphates emerged besides faint iron oxides peaks when dihydrogen phosphates used as hydrogen donors. The formation of iron phosphates might be attributed to the reaction of phosphates with $\mathrm{Fe}^{0}$ or released iron from initial corrosion of $\mathrm{Fe}^{0}$ $[22,23]$. It indicates that the phosphates might compete for reactive sites on nanoscale particle surface with water and conse- quently affect the PCB dechlorination. Results of EDX analysis in Fig. $2 \mathrm{~b}$ and $\mathrm{c}$ show that the Ni percentage at the outer layer of particles after reaction decreased with increase of amount of organic acid used as compared to that without hydrogen donor. This suggests that partial dislodgement of $\mathrm{Ni}$ from bimetallic Ni/Fe might occur due to the dissolution of Fe into liquid phase especially in the presence of organic acid $[10,11]$, which could impair the catalytic function of $\mathrm{Ni} / \mathrm{Fe}$ particles.

\subsection{Dechlorination products}

Fig. 3 shows the GC-MS chromatograms of the raw capacitor oil and products after reaction with and without hydrogen donors, respectively. Fig. 3g-i are the magnification of Fig. 3f, d, and e with the purpose of convenient description of degradation products. Fig. 3a indicates that all revealed PCB congeners comprised of different positional isomers, including ortho-, para- and meta-chlorine substituted PCBs. As compared to GC-MS profile of products without hydrogen donor in Fig. 3f, oxalic acid and acetic acid caused significant dechlorination shown in Fig. $3 d$ and e but only trace amounts of new mon- and dichlorobiphenyls were observed as dechlorination products in the presence of dihydrogen phosphates shown in Fig. 3b and c.

Positive dechlorination products mainly consisted of 1-alkylbenzenes along with a little biphenyl and cyclohexyl-benzene in the presence of organic acids in Fig. 3h and i. Besides these positive dechlorination products, alkanes also emerged as products in Fig. $3 \mathrm{~h}$ and i. The appearance of these alkanes might be ascribed to the further reductive hydrogenation of 1-alkyl-benzenes because no unsaturated hydrocarbons are observed in raw capacitor oil as shown in Fig. 3a. After reaction without hydrogen donor, however, dechlorination products were mainly composed of biphenyl, 1-alkyl-benzenes and other lower chlorinated biphenyls in Fig. 3g. However, there were little alkanes observed as degradation products in the absence hydrogen donor, which suggests that further hydrogenation of 1-alkyl-benzenes was mainly ascribed to the employment of organic acid as hydrogen donor. The notable difference in distribution of dechlorination products among different hydrogen donors shows that the $\mathrm{H}^{+}$from dissociation of oxalic acid and acetic acid could play similar role as $\mathrm{H}^{+}$from self-dissociation of $\mathrm{H}_{2} \mathrm{O}$ and be used for dechlorination in subcritical water. However, $\mathrm{H}^{+}$from dihydrogen phosphates could not counterbalance the negative effects of $\mathrm{PO}_{4}^{3-}$ on reactivity of nanoscale $\mathrm{Ni} / \mathrm{Fe}$ towards to PCB dechlorination, which resulted in inferior dechlorination performance.

\subsection{Optimization of dechlorination parameters}

\subsubsection{Effects of different hydrogen donors}

Fig. 4 compares the total $\mathrm{PCB}$ conversion ratio in the presence of $\mathrm{NaH}_{2} \mathrm{PO}_{4}, \mathrm{KH}_{2} \mathrm{PO}_{4}, \mathrm{HAc}$ and oxalic acid, respectively. Taking into account the different dissociation constant, mass of each donor used was calculated according to the same amount of $\mathrm{H}^{+}$dissociated from each donor based on dissociation constant. Total conversion ratio decreased with increasing mass of hydrogen donor in all cases. As shown in Fig. 4c and d, PCB conversion ratio of $83.4 \%$ with $0.5 \mathrm{mg}$ oxalic acid was almost same as that $82.8 \%$ with $1 \mathrm{mg}$ oxalic acid, but the same PCB conversion ratio of $82.0 \%$ with $1 \mathrm{mg}$ acetic acid and $81.37 \%$ with $5 \mathrm{mg}$ acetic acid were superior to that $75.64 \%$ with $10 \mathrm{mg}$ acetic acid. Accordingly, 0.5-1 mg of oxalic acid and 1$5 \mathrm{mg}$ of HAc can be regarded as optimal mass range used as hydrogen donor for PCB dechlorination respectively under this experimental condition. Taking into account the statistical error and cost of each hydrogen donor, addition of hydrogen donors enhanced PCB dechlorination with an increasing order of $\mathrm{KH}_{2} \mathrm{PO}_{4} \approx$ $\mathrm{NaH}_{2} \mathrm{PO}_{4}<\mathrm{HAc} \approx$ oxalic acid. 

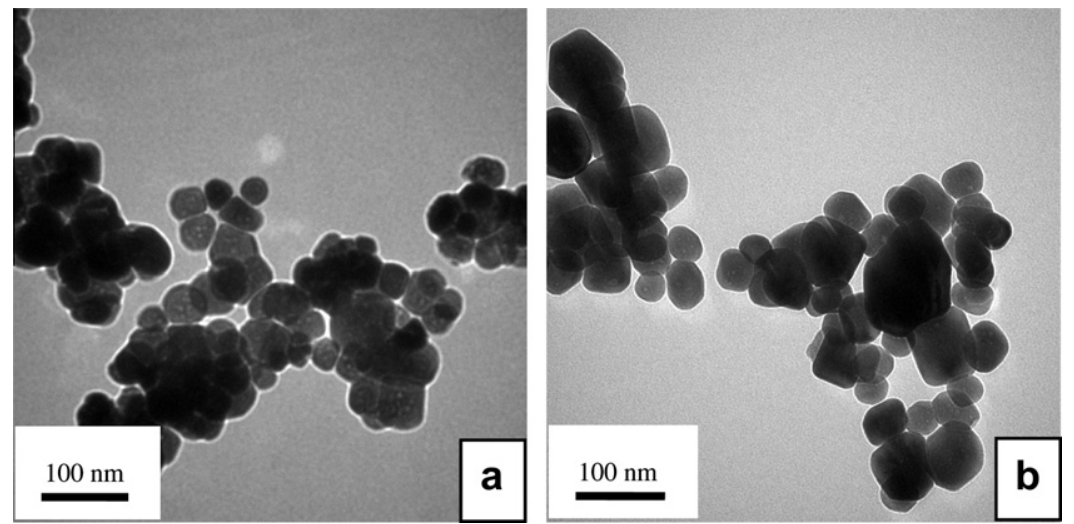

Fig. 1. Transmission electron microscope (TEM) images of (a) fresh Ni/Fe particles and (b) spent Ni/Fe particles after reaction used oxalic acid as hydrogen donor.
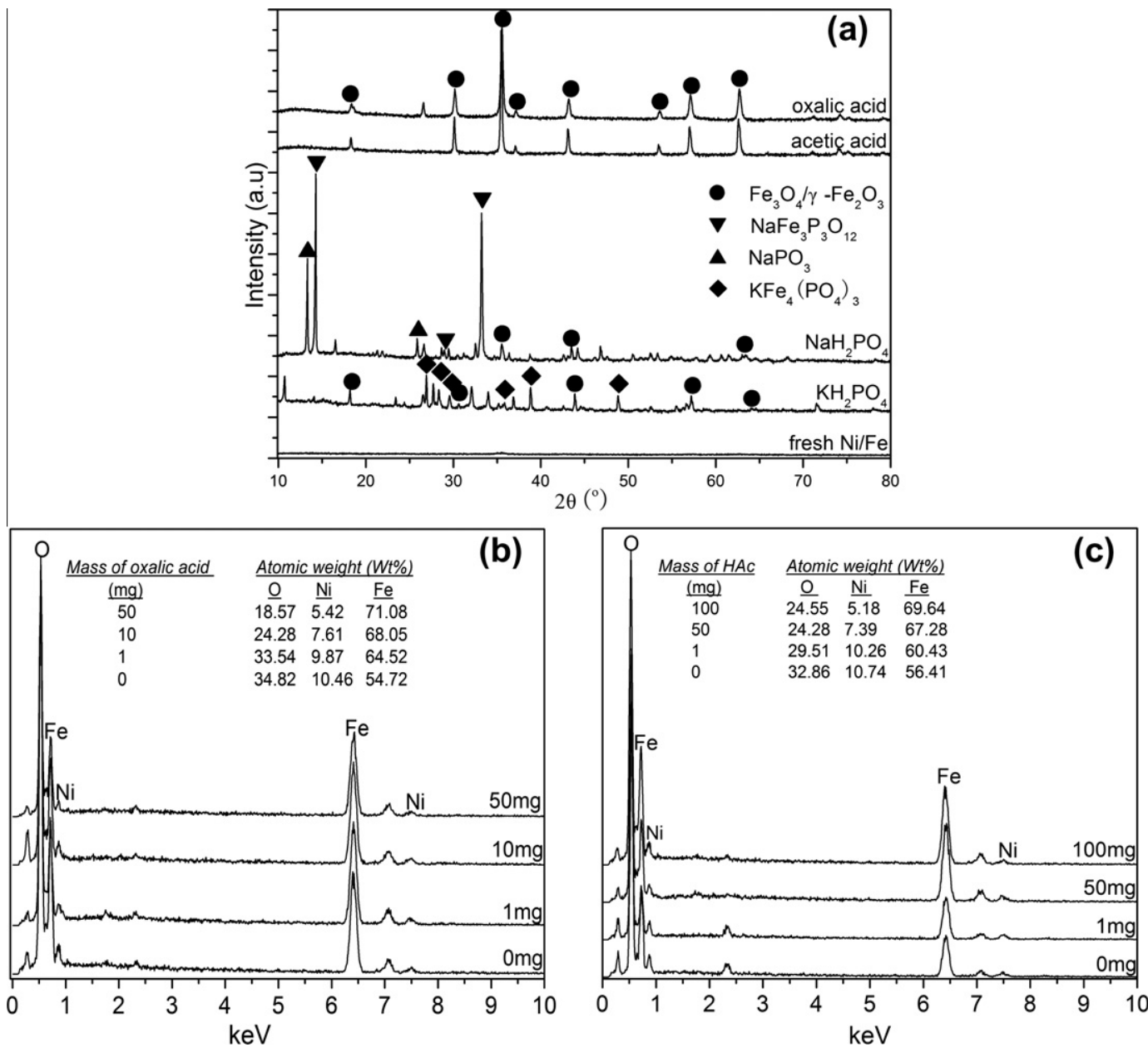

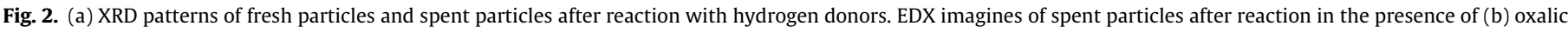
acid, and (c) HAc. PCBs concentration in water $C_{\mathrm{PCBs}}=5.25 \mathrm{mg} / \mathrm{L}$, dosage of Ni/Fe particles in water $C_{\mathrm{Ni} / \mathrm{Fe}(\mathrm{s})}=6.25 \mathrm{~g} / \mathrm{L}, T=330{ }^{\circ} \mathrm{C}, t=3 \mathrm{~h}$.

Previous studies reported that enhanced dechlorination in the presence of sodium hypophosphite was attributed to the formation of reductive hydrogen gas via sodium hypophosphite decomposition $[24,25]$. However, dihydrogen phosphates used in this study could not be decomposed to form hydrogen gas but supply $\mathrm{H}^{+}$for corrosion of $\mathrm{Fe}^{0}$ to generate hydrogen species which subsequently were used to hydrodechlorination. Although the $\mathrm{pH}(<5.5)$ of water was lower than that maximum of pure subcritical water $(\mathrm{pH} \approx 5.5)$ [26] due to the addition of dihydrogen phosphates, the total conver- sion ratios for both dihydrogen phosphates were below $65 \%$ which is lower than that $(>75 \%)$ achieved in pure subcritical water without hydrogen donor [4]. The inferior dechlorination efficiency for both dihydrogen phosphates was mainly attributed to the complex precipitation of iron phosphates on particles surface as shown in Fig. 2a. It has been demonstrated that phosphates could react with reactive functional groups on the zerovalent iron (ZVI) surface or released iron to form insoluble inner-sphere complexes on particle surface, which then caused the suspension of dechlorination 

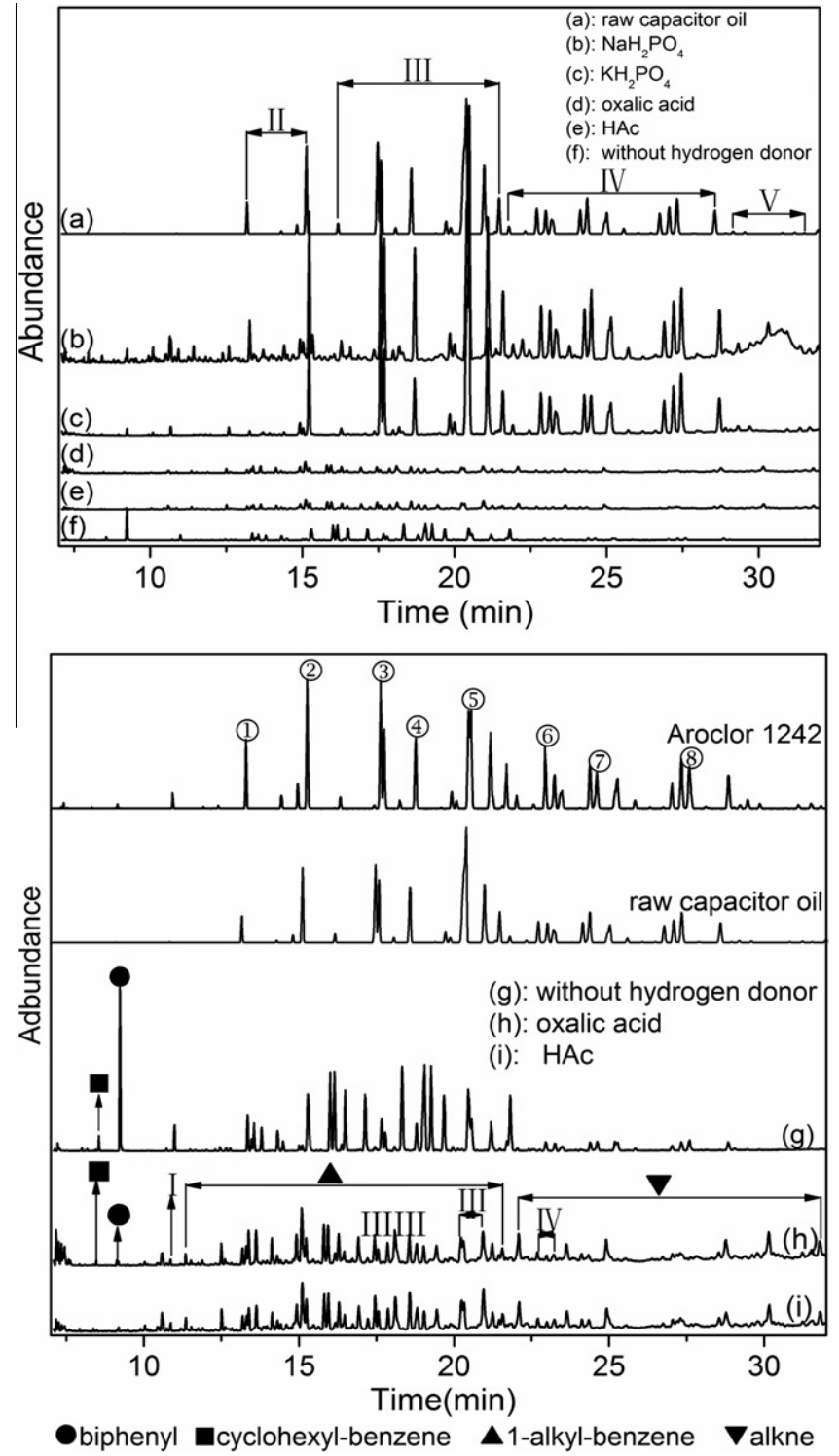

Fig. 3. GC-MS chromatograms of (a) raw capacitor oil and products after reaction with hydrogen donors, (b) $\mathrm{NaH}_{2} \mathrm{PO}_{4}$, (c) $\mathrm{KH}_{2} \mathrm{PO}_{4}$, (d) oxalic acid, (e) HAc and (f) without hydrogen donor. (g-i) are enlargements of (f), (d) and (e). Peaks of I-V were identified as follows: $\mathrm{I}$ = monochlorobiphenyl; II = dichlorobiphenyl; III = trichlorobhenyl; IV = tetrachlorobhenyl; $\mathrm{V}=$ pentachlorophenyl. $C_{\mathrm{PCBs}}=5.25 \mathrm{mg} / \mathrm{L}, C_{\mathrm{Ni} / \mathrm{Fe}(\mathrm{s})}$ $=6.25 \mathrm{~g} / \mathrm{L}, T=330^{\circ} \mathrm{C}, t=3 \mathrm{~h}$.

significantly in ambient water $[22,23,27]$. In present batch tests, higher reaction temperature $\left(>250^{\circ} \mathrm{C}\right)$ as compared to ambient water could speed up the reaction of phosphates with functional groups considerably, and hence resulted in the rapid formation of insoluble iron phosphates. These insoluble iron phosphates precipitated and blocked the reactive site on particles surface, and consequently caused the suspension of $\mathrm{PCB}$ dechlorination significantly. Although the concentration of $\mathrm{H}^{+}$increased with the mass of phosphates added, the increase of amount of $\mathrm{PO}_{4}^{3-}$ caused more rapid formation of iron phosphates which were insoluble even at lower $\mathrm{pH}$. Hence, the particles were quickly covered by complex precipitates following the drop of dechlorination efficiency.

Acids, regardless of whether it is organic or inorganic, not only play the role of reaction enhancer by supplying $\mathrm{H}^{+}$for generation of hydrogen species but also act as reaction retarder in point of causing the corrosive dislodgment of catalyst from bimetallic particles $[11,28]$. The results in Fig. $4 \mathrm{c}$ and d indicate that water under weak acid condition favored the hydrodechlorination at subcritical state. Schrick et al. [29] found nickel and iron needed to be in electronic contact in order to facilitate the catalysis of hydrogen species to generate active hydrogen species. In weak acidic water, the amount of $\mathrm{H}^{+}$is sufficient to produce hydrogen species via corrosion of $\mathrm{Fe}^{0}$ but not enough to cause the break of electronic contact between $\mathrm{Fe}^{0}$ and $\mathrm{Ni}^{\mathrm{O}}$ as shown in Fig. 1b, which was also observed by Bokare et al. $[10,11]$. Meantime, lower $\mathrm{pH}$ caused by the addition of organic acid could prevent formation of passivation layer to a certain extent in ambient water [13]. Unlike to $\mathrm{PO}_{4}^{3-}, \mathrm{CH}_{3} \mathrm{COO}^{-}$and ${ }^{-} \mathrm{OOC}-\mathrm{COO}^{-}$ could not react with released iron to form iron oxalates or iron acetates but form iron oxides as passivation layer, which is confirmed by XRD patterns of spent particles in Fig. 2a. Increase of amount of organic acid could promote the corrosion of $\mathrm{Fe}^{0}$ significantly as well as the increase of production of hydrogen species, but the electronic contact between $\mathrm{Fe}^{0}$ and $\mathrm{Ni}^{\circ}$ was also damaged due to the serious corrosion of $\mathrm{Fe}^{0}$. Subsequently, the partial $\mathrm{Ni}$ was dislodged from surface of integrated $\mathrm{Ni} / \mathrm{Fe}$ particles which was confirmed by the decrease of $\mathrm{Ni}$ percentage in $\mathrm{Ni} / \mathrm{Fe}$ particles as increase of organic acids added shown in Fig. 2b and $\mathrm{c}$ as EDX results of spent particles after reaction with organic acids. Therefore, the catalytic function of $\mathrm{Ni} /$ Fe was impaired, which caused that the bimetallic particles were disabled from catalysis to generate reactive hydrogen species following the drop of dechlorination efficiency.

\subsubsection{Effect of reaction time}

Due to the remarkable retardant effects of dihydrogen phosphates on dechlorination, acetic acid and oxalic acid were chose for investigating the effects of reaction time on dechlorination at $330{ }^{\circ} \mathrm{C}$ without residence at low temperature. As shown in Fig. 5, total conversion ratio increased with time but appeared a plateau after $3 \mathrm{~h}$ for all cases. Compared results demonstrate that little addition of organic acid as hydrogen donor could facilitate dechlorination notably. In terms of optimal amount used, the enhanced dechlorination efficiency caused by oxalic acid is almost equivalent to that caused by acetic acid, which is also indicated in Fig. 4c and $\mathrm{d}$. As prolonging of reaction time, the corrosion of $\mathrm{Fe}^{0}$ proceeded fast and then slowed down along with release of iron and generation of active hydrogen species for catalytic hydrodechlorination of PCBs. During this process, the released iron reacted with hydroxyl to form insoluble iron oxides (Fig. 2a) which then precipitated on particle surface and covered the reactive sites. After all fresh particle surfaces were covered by insoluble precipitates, the corrosion of $\mathrm{Fe}^{0}$ ceased and thereby dechlorination slowed down to termination with time, which is confirmed by the appearance of plateau after $3 \mathrm{~h}$ in Fig. 5. This indicates that higher dechlorination efficiency could not be achieved even with considerable increase of reaction time of dechlorination at $330^{\circ} \mathrm{C}$ despite the addition of organic acid. Although the reactivity of passivation bimetallic particles could be partially restored via $\mathrm{HCl}$ wash for eliminating the oxides layer on particle surface [21], retard of the passivating rate during dechlorination process is more economical in terms of the operation cost of $\mathrm{HCl}$ wash after reaction. It is undoubted that residence at low temperature for a moment can be regarded as a favorite alternative to retard passivation during dechlorination process.

\subsubsection{Effect of temperature at first stage}

In this section, the whole dechlorination process was divided into two stage in terms of reaction temperature: first stage for corrosion of $\mathrm{Fe}^{0}$ at low temperature $\left(<250^{\circ} \mathrm{C}\right)$ and subsequent second stage for PCB dechlorination at $330^{\circ} \mathrm{C}$. Fig. 6 presents the effects of temperature at first stage on whole dechlorination and corrosion of selected metals with residence at second stage for $1 \mathrm{~h}$. As shown in Fig. 6a, total conversion ratio increased with decline of temperature at first stage. With $1 \mathrm{mg}$ oxalic acid, the total conversion ratio 

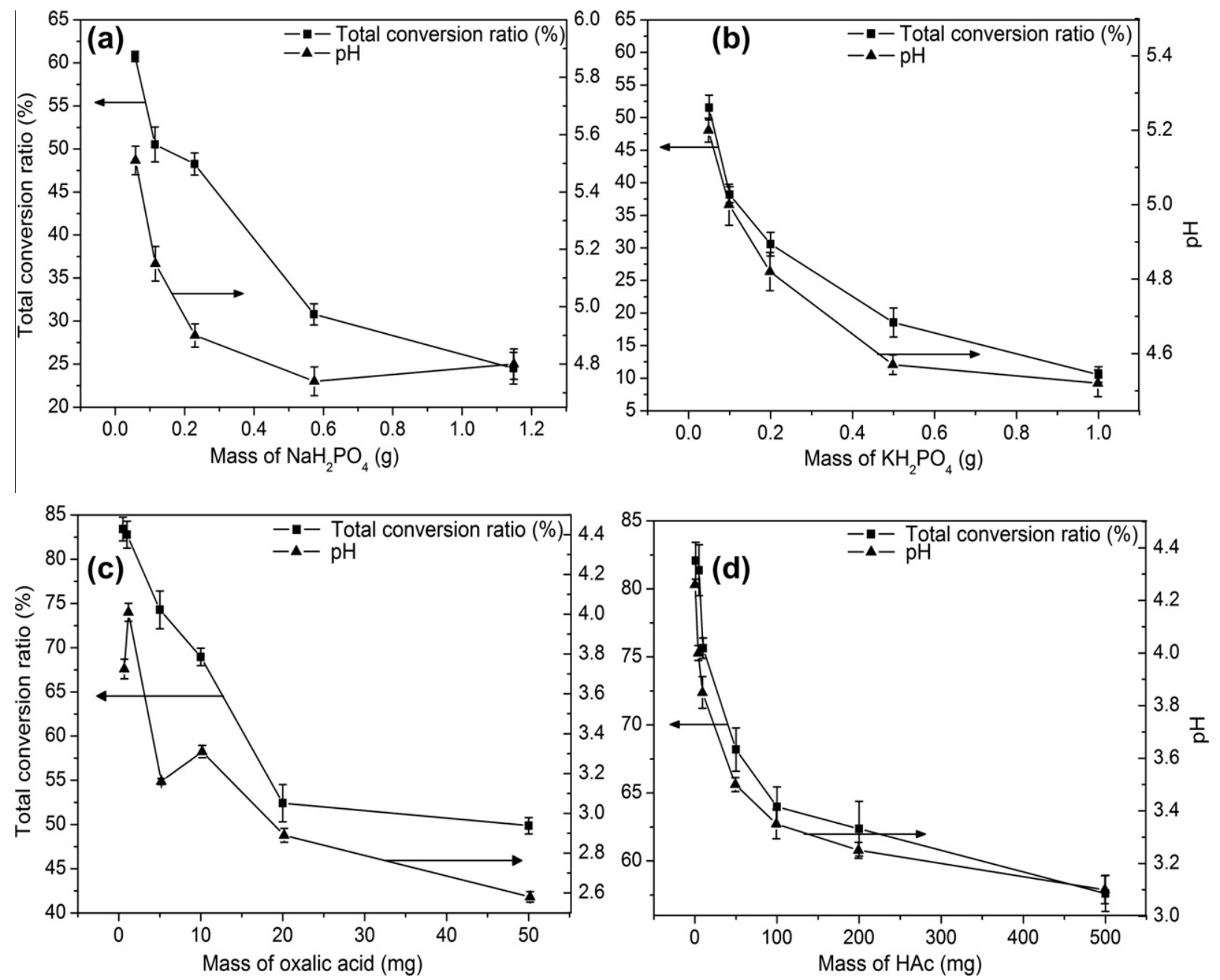

Fig. 4. Effect of mass of hydrogen donor (a) $\mathrm{NaH}_{2} \mathrm{PO}_{4}$, (b) $\mathrm{KH}_{2} \mathrm{PO}_{4}$, (c) oxalic acid, (d) $\mathrm{HAc}$ on dechlorination. $C_{\mathrm{PCBs}}=5.25 \mathrm{mg} / \mathrm{L}, C_{\mathrm{Ni} / \mathrm{Fe}(\mathrm{s})}=6.25 \mathrm{~g} / \mathrm{L}, T=330{ }^{\circ} \mathrm{C}, t=3 \mathrm{~h}$.

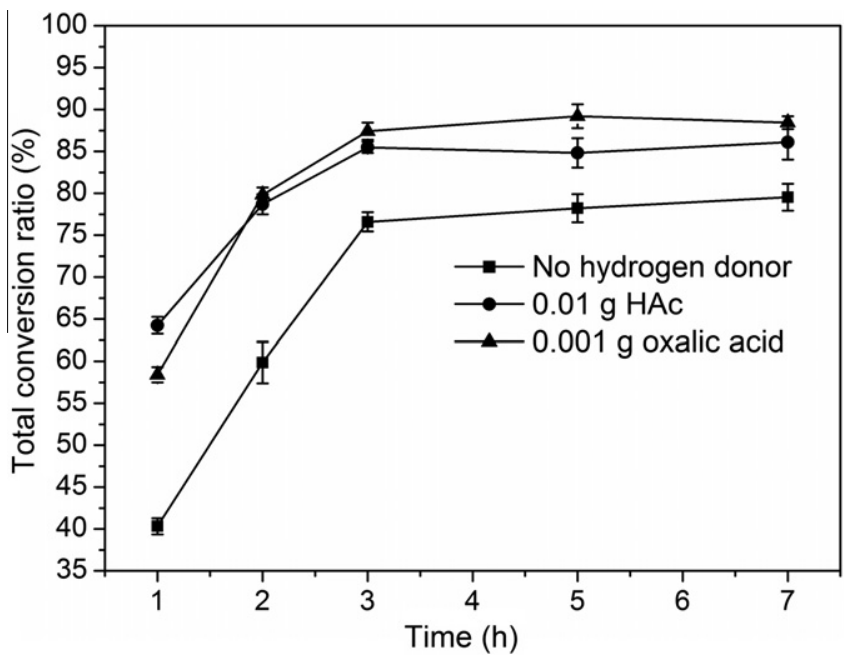

Fig. 5. Effect of reaction time on dechlorination in the presence of oxalic acid and HAc. $C_{\mathrm{PCBs}}=5.25 \mathrm{mg} / \mathrm{L}, C_{\mathrm{Ni} / \mathrm{Fe}(\mathrm{s})}=6.25 \mathrm{~g} / \mathrm{L}, T=330^{\circ} \mathrm{C}$.

for $150{ }^{\circ} \mathrm{C}$ and $200{ }^{\circ} \mathrm{C}$ are $89.7 \%$ and $82.6 \%$, respectively, which were superior or equal to that in Fig. 5 without residence at first stage. This indicates that lower temperature at first stage might favor the following dechlorination at $330{ }^{\circ} \mathrm{C}$. Apparently, the corro- sion and passivation of $\mathrm{Ni} / \mathrm{Fe}$ particles would be completed rapidly in subcritical water owing to the high temperature above $250{ }^{\circ} \mathrm{C}$ in previous reports $[2,3]$. Low temperature at first stage in this study might retard the corrosion of $\mathrm{Fe}^{0}$ and formation of insoluble iron oxides, which hence enhanced the utility efficiency of $\mathrm{Fe}^{0}$ and yield of hydrogen species used for dechlorination. Total conversion ratios for both $150{ }^{\circ} \mathrm{C}$ and $200{ }^{\circ} \mathrm{C}$ dropped drastically when the amount of oxalic acid was beyond $2 \mathrm{mg}$. This might be resulted from the corrosive dislodgment of $\mathrm{Ni}$ from $\mathrm{Ni} / \mathrm{Fe}$ particles due to the strong acidity with $2 \mathrm{mg}$ or more oxalic acid added into water $(\mathrm{pH}<4.1)$.

$\mathrm{Ni}, \mathrm{Cr}, \mathrm{Cu}, \mathrm{Fe}, \mathrm{Mn}$ were chose as preferred monitored metals in respect that they were the main composition elements of constructed materials of the autoclave as well as constitutes of bimetallic particles. Apparently, the concentration of all monitored metal ions except for $\mathrm{Fe}$ in aqueous phase after reaction was almost constant regardless of addition of oxalic acid in Fig. $6 \mathrm{~b}$ and c. Iron concentration increased as the increase of oxalic acid amount to $1 \mathrm{mg}$, after that, it declined with the increase of amount to $3 \mathrm{mg}$, and then increased again. The change tendency of iron concentration both in Fig. $6 \mathrm{~b}$ and $\mathrm{c}$ shows that the affect of temperature on corrosion of iron was sharper than that on other metals. It should be noted that iron and nickel ions in the aqueous phase after reaction were the combined results of the corrosion of $\mathrm{Ni} / \mathrm{Fe}$ particles and constructed materials of the autoclave. As compared to $\mathrm{Fe}$, however, element $\mathrm{Ni}$ is more resistant to acid corrosion even 

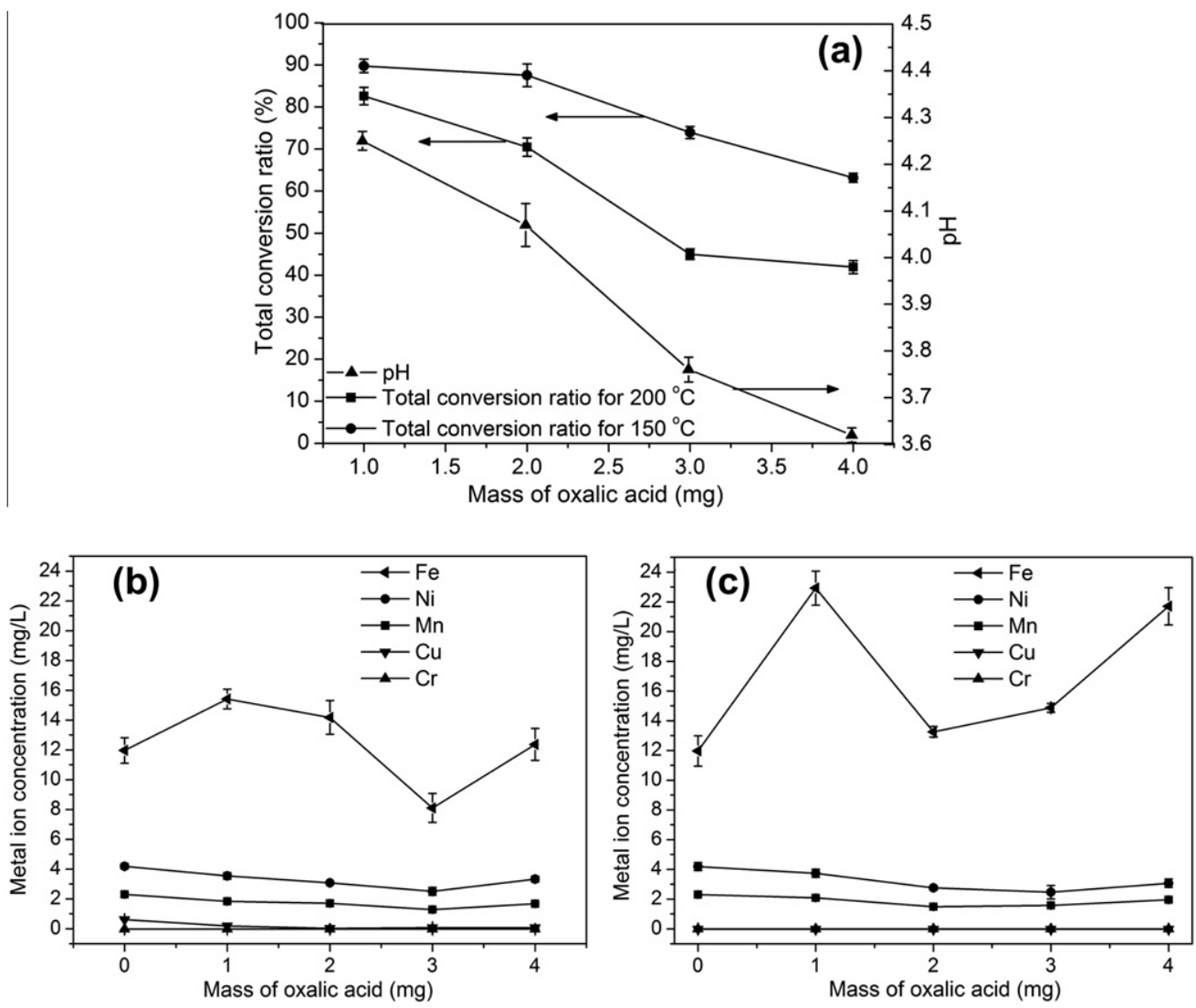

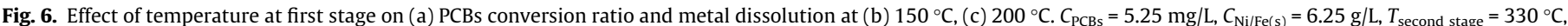
$t_{\text {first stage }}=1 \mathrm{~h}, t_{\text {second stage }}=3 \mathrm{~h}$.

in subcritical water according to the metal activity order, which resulted that the strong acid corrosion in acid solution mainly occurred on Fe surface other than Ni surface although theses two metals contact closely in $\mathrm{Ni} / \mathrm{Fe}$. Therefore, the dissolution of $\mathrm{Fe}$ was predominant in subcritical water in the presence of organic acid, which resulted that iron ions were main composition in aqueous phase after reaction. But $\mathrm{Ni}$ almost kept stable during whole reaction even in the presence of organic acid and hence the leaching of Ni could hardly be observed with the increase of acid mass added. Meantime, nickel was also prevented from corrosion via cathodic protection by $\mathrm{Fe}^{0}$ in bimetallic particles, which was also observed in previous report [29]. As for other monitored metals, such as $\mathrm{Cr}, \mathrm{Cu}, \mathrm{Mn}$, their resistance to acid leaching were mainly ascribed to their alloy state existed in constructed materials of the autoclave. As compared to the high reactive nanoscale $\mathrm{Ni} / \mathrm{Fe}$, these alloy metals could hardly compete for $\mathrm{H}^{+}$with $\mathrm{Ni} / \mathrm{Fe}$ and hence were protected from acid corrosion. Therefore, the high concentration of iron ions in aqueous phase after reaction was mainly resulted from the corrosion of $\mathrm{Fe}^{0}$ of $\mathrm{Ni} / \mathrm{Fe}$ particles, which also indirectly confirmed that addition of oxalic acid could enhance utility efficiency of $\mathrm{Fe}^{0}$. Considering the dechlorination performance and corrosion of $\mathrm{Ni} / \mathrm{Fe}, 150{ }^{\circ} \mathrm{C}$ was regarded as optimal temperature adopted at first stage.

\subsubsection{Development of the two-stage process}

In order to optimize the whole process, $150{ }^{\circ} \mathrm{C}$ was chose as optimum temperature for $\mathrm{Fe}^{0}$ corrosion at first stage on the basis of results in Fig. 6 and $330^{\circ} \mathrm{C}$ was still regarded as optimum temperature for PCB dechlorination according to our previous report [4]. Fig. 7 shows the change tendency of the total conversion ratio with residency time at different stage. Apparently, total conversion ratio increased gradually with time at first stage under the same residency time at second stage. And total conversion ratios for all batch experiments conducted at second stage for $2 \mathrm{~h}$ were greater than that only conducted at second stage for $3 \mathrm{~h}$, indicating that $\mathrm{Fe}^{0}$ corrosion at first stage could promote dechlorination. It should be noted that the conversion ratio with only residency at first stage for $3 \mathrm{~h}$ is $46.5 \%$ (Fig. 7). The remarkable difference in dechlorination efficiency with and without residency at second stage shows that the main dechlorination was achieved at the second stage under

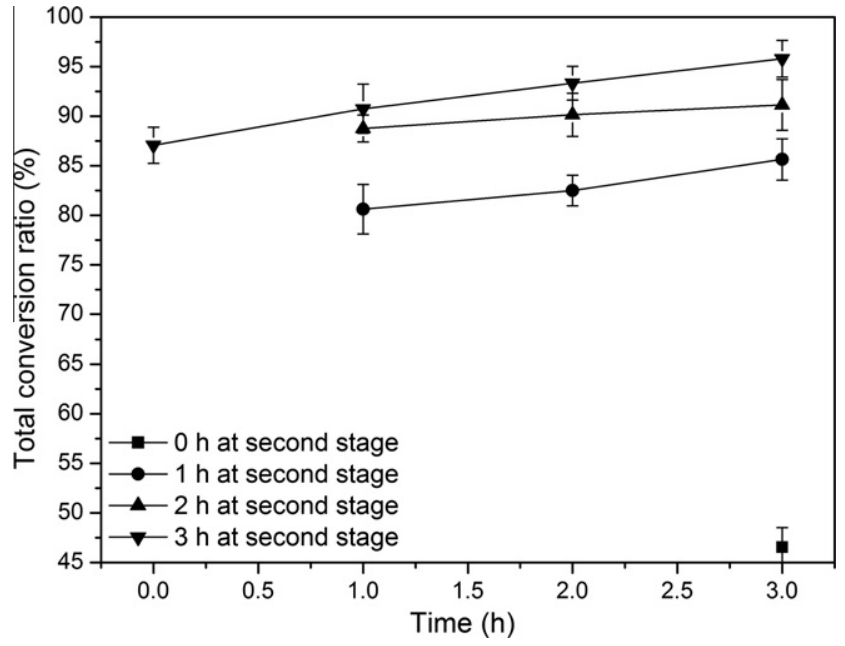

Fig. 7. Effect of residence time on dechlorination during two-stage reaction process $X$ axis: residence time at first stag, $C_{\mathrm{PCBs}}=5.25 \mathrm{mg} / \mathrm{L}, C_{\mathrm{Ni} / \mathrm{Fe}(\mathrm{s})}=6.25 \mathrm{~g} / \mathrm{L}, T_{\text {first stage }}$ $=150{ }^{\circ} \mathrm{C}, T_{\text {second stage }}=330^{\circ} \mathrm{C}$. 
$330^{\circ} \mathrm{C}$. The first stage was mainly used for conducting $\mathrm{Fe}^{0}$ corrosion and generation of hydrogen species. It has been reported that the relative resistance of positional isomers increased from para- to meta- to ortho-positions, which resulted in chlorines in the paraand meta-position were predominantly removed over chlorines in the ortho-position during the dechlorination process [7,30-33]. Therefore the total conversion ratio of $46.5 \%$ with only residency at first stage in Fig. 7 might be attributed to the removal of chlorines in the para- or meta-position attached to biphenyl rings. It is well known that the solubility of hydrogen species generated from corrosion of $\mathrm{Fe}^{0}$ increased with the decrease of temperature in hot water, thereby $150{ }^{\circ} \mathrm{C}$ at first stage might conduce to more dissolved hydrogen species in comparison to that at second stage under $330^{\circ} \mathrm{C}$. Since the catalysis of hydrogen species to form active hydrogen species happened on the surface of catalytic metals $[10,34]$, more dissolved hydrogen species could result that more hydrogen species were adsorbed on $\mathrm{Ni}^{0}$ surface and then catalyzed to form active hydrogen species. The active hydrogen species generated at first stage were then used to hydrodechlorinate the PCBs at second stage.

\section{Conclusions}

The results of this study demonstrate that the PCB dechlorination efficiency depends strongly on the property of hydrogen donors in subcritical water. The anion introduced along with the hydrogen donor plays a key role in overall dechlorination process. Phosphates inhibit the dechlorination remarkably via undesired competitive reaction with $\mathrm{Ni} / \mathrm{Fe}$ to form thick passivation layer on particle surface. In contrast, oxalic and acetic anions cannot compete for reactive nanoscale $\mathrm{Ni} / \mathrm{Fe}$ with $\mathrm{H}^{+}$. Meanwhile, the two-stage process combined with addition of oxalic acid can enhance utility efficiency of $\mathrm{Fe}^{0}$ and yield of active hydrogen species such as atomic $\mathrm{H}^{*}$ and metal hydride $\mathrm{Ni}-\mathrm{H}$, thereby improve subsequent $\mathrm{PCB}$ dechlorination significantly. The obtained conclusions also pose a precaution that it is necessary to perform $\mathrm{pH}$ adjustment and desalination when dehalogenation using nanoscale iron-based particles is carried out in subcritical water.

\section{Acknowledgements}

This work was made possible by the financial supports from the National Key Technology R \& D Program (2008BAC32B03), the National Natural Science Foundation of China (21077120) and the National Water Pollution Control and Management Program (2009ZX07212-002).

\section{References}

[1] A. Kubátová, J. Herman, T.S. Steckler, M.D. Veij, D.J. Miller, E.B. Klunder, C.M. Wai, S.B. Hawthorne, Subcritical (hot/liquid) water dechlorination of PCBs (Aroclor1254) with metal additives and in waste paint, Environ. Sci. Technol. 37 (2003) 5757-5762.

[2] N. Kluyev, A. Cheleptchikov, E. Brodsky, V. Soyfer, V. Zhilnikov, Reductive dechlorination of polychlorinated dibenzo-p-dioxins by zerovalent iron in subcritical water, Chemosphere 46 (2002) 1293-1296.

[3] H. Hori, Y. Nagaoka, A. Yamamoto, T. Sano, N. Yamashita, S. Taniyasu, S. Kutsuna, Efficient decomposition of environmentally persistent perfluorooctanesulfonate and related fluorochemicals using zerovalent iron in subcritical water, Environ. Sci. Technol. 40 (2006) 1049-1054.

[4] N.M. Zhu, Y. Li, F.S. Zhang, Catalytic dechlorination of polychlorinated biphenyls in subcritical water by Ni/Fe nanoparticles, Chem. Eng. J. 171 (2011) 919-925.

[5] C.F. Wang, N.N. Zhu, Y.M. Wang, F.S. Zhang, Co-detoxification of transformer oil contained PCBs and heavy metals in medical waste incinerator fly ash under sub- and supercritical water, Environ. Sci. Technol. 46 (2012) 1003-1009.

[6] H. Choi, S.R. Al-abed, S. Agarwal, Effects of aging and oxidation of palladized iron embedded in activated carbon on the dechlorination of 2-chlorobiphenyl, Environ. Sci. Technol. 43 (2009) 4137-4142.
[7] Y. Zhuang, S.W. Ahn, R.G. Luthy, Debromination of polybrominated diphenyl ethers by nanoscale zerovalent iron: pathways, kinetics, and reactivity, Environ. Sci. Technol. 44 (2010) 8236-8242.

[8] F. He, D.Y. Zhao, Preparation and characterization of a new class of starchstabilized bimetallic nanoparticles for degradation of chlorinated hydrocarbons in water, Environ. Sci. Technol. 39 (2005) 3314-3320.

[9] Y.H. Shih, Y.T. Tai, Reaction of decabrominated diphenyl ether by zerovalen iron nanoparticles, Chemosphere 78 (2010) 122-1206

[10] A.D. Bokare, R.C. Chikate, C.V. Rode, K.M. Paknikar, Iron-nickel bimetallic nanoparticles for reductive degradation of azo dye Orange $G$ in aqueous solution, Appl. Catal. B: Environ. 79 (2008) 270-278.

[11] J.J. Wei, X.H. Xu, Y. Liu, D.H. Wang, Catalytic hydrodechlorination of 2,4 dichlorophenol over nanoscale $\mathrm{Pd} / \mathrm{Fe}$ : reaction pathway and some experimental parameters, Water Res. 40 (2006) 348-354.

12] H. Tian, J.J. Li, Z. Mu, L.D. Li, Z.P. Hao, Effects of pH on DDT degradation in aqueous solution using bimetallic Ni/Fe nanoparticles, Sep. Purif. Technol. 66 (2009) 84-89.

[13] X.H. Xu, J.J. Wo, J.H. Zhang, Y.J. Wu, Y. Liu, Catalytic dechlorination of $p$-NCB in water by nanoscale Ni/Fe, Desalination 242 (2009) 346-354.

[14] A. Kubátová, A.J.M. Lagadec, S.B. Hawthorne, Dechlorination of lidane, dieldrin, tetrachloroethane, trichloroethene, and PVC in subcritical water, Environ. Sci. Technol. 36 (2002) 1337-1343.

[15] United States Environmental Protection Agency, Test Method, EPASW-846 on line, 2009. <http://www.epa.gov/epawaste/hazard/testmethods/sw846/ online/>.

[16] M. Takada, H. Toda, R. Uchida, A new rapid method for quantification of PCBs in transformer oil, Chemosphere 43 (2001) 455-459.

[17] H.A.R. Martínez, G.C. Rodríguez, D.H. Castillo, Determination of PCBs in transformation oil using gas chromatography with mass spectroscopy and Aroclors (A1254:A1260), J. Mex. Chem. Soc. 49 (2005) 263-270.

[18] G.M. Frame, R.E. Wagner, J.C. Carnahan, J.F. Brown Jr., R.J. May, L.A. Smullen, D.L. Bedard, Comprehensive, quantitative, congener-specific analysis of eight Aroclors and complete PCB congener assignments on DB-1 capillary GC columns, Chemosphere 33 (1996) 603-623.

[19] J. Feng, T.T. Lim, Pathways and kinetics of carbon tetrachloride and chloroform reductions by nano-scale $\mathrm{Fe}$ and $\mathrm{Fe} / \mathrm{Ni}$ particles: comparison with commercial micro-scale Fe and Zn, Chemosphere 59 (2005) 1267-1277.

[20] J.E. Martin, A.A. Herzing, W.L. Yan, X.Q. Li, B.E. Koel, C.J. Kiely, W.X. Zhang, Determination of the oxide layer thickness in core-shell zerovalent iron nanoparticles, Langmuir 24 (2008) 4329-4334.

[21] B.W. Zhu, T.T. Lim, Catalytic reduction of chlorobenzenes with $\mathrm{Pd} / \mathrm{Fe}$ nanoparticles: reactive sites, catalyst stability, particle aging, and regeneration, Environ. Sci. Technol. 41 (2007) 7523-7529.

[22] T.T. Lim, B.W. Zhu, Effects of anions on the kinetics and reactivity of nanoscale $\mathrm{Pd} / \mathrm{Fe}$ in trichlorobenzene dechlorination, Chemosphere 73 (2008) 1471-1477.

[23] C.M. Su, R.W. Plus, Arsenate and arsenite removal by zerovalent iron: effects of phosphate, silicate, carbonate, borate, sulfate, chromate, molybdate, and nitrate, relative to chloride, Environ. Sci. Technol. 35 (2001) 4562-4568.

[24] H. Hidaka, A. Saitou, H. Honjou, K. Hosada, M. Moriya, N. Serpone, Microwaveassisted dechlorination of polychlorobenzenes by hypophosphite anions in aqueous alkaline media in the presence of Pd-loaded active carbon, J. Hazard. Mater. 148 (2007) 22-28.

[25] X.T. Liu, Q. Zhang, G.X. Zhang, R. Wang, Application of microwave irradiation in the removal of polychlorinated biphenyls from soil contaminated by capacitor oil, Chemosphere 72 (2008) 1655-1658.

[26] A. Kruse, E. Dinjus, Hot compressed water as reaction medium and reactant: properties and synthesis reactions, J. Supercrit. Fluids 39 (2007) 362-380.

[27] Y. Liu, T. Phenrat, G.V. Lowry, Effects of TCE concentration and dissolved groundwater soluted on NZVI-promoted TCE dechlorination and $\mathrm{H}_{2}$ evolution, Environ. Sci. Technol. 41 (2007) 7881-7887.

28] N. He, PJ. Li, Y.C. Zhou, W.X. Ren, S.X. Fan, V.A Verkhozina, Catalytic dechlorination of polychlorinated biphenyls in soil by palladium-iron bimetallic catalyst, J. Hazard. Mater. 164 (2009) 126-132.

[29] B. Schrick, J.L. Blough, A.D. Jones, T.E. Mallouk, Hydrodechlorination of trichloroethylene to hydrocarbons using bimetallic nickel-iron nanoparticles, Chem. Mater. 14 (2002) 5140-5147.

[30] H.K. Yak, Q.Y. Lang, C.M. Wai, Relative resistance of positional isomers of polychlorinated biphenyls toward reductive dechlorination by zerovalent iron in subcritical water, Environ. Sci. Technol. 34 (2000) 2792-2798.

[31] G.V. Lowry, K.M. Johnson, Congener-specific dechlorination of dissolved PCBs by microscale and nanoscale zerovalent iron in a water/methanol solution, Environ. Sci. Technol. 38 (2004) 5208-5216.

[32] S. Agarwal, S.R. Al-abed, D.D. Dionysiou, E. Graybill, Reactivity of substituted chlorines and ensuing dechlorination pathways of select PCB congeners with Pd/Mg bimetallics, Environ. Sci. Technol. 43 (2009) 915-921.

[33] H. Choi, S.R. Al-abed, S. Agarwal, Catalytic role of palladium and relative reactivity of substituted chlorines during adsorption and treatment of PCBs on reactive activated carbon, Environ. Sci. Technol. 43 (2009) 7510-7515.

[34] Y. Han, W. Li, M.H. Zhang, K.Y. Tao, Catalytic dechlorination of monochlorobenzene with a new type of nanoscale $\mathrm{Ni}(\mathrm{B}) / \mathrm{Fe}(\mathrm{B})$ bimetallic catalytic reductant, Chemosphere 72 (2008) 53-58. 